\title{
Experimental Study of Linear Closed-Loop Control of Subsonic Cavity Flow
}

\author{
P. Yan, ${ }^{*}$ M. Debiasi,${ }^{\dagger}$ X. Yuan,${ }^{\ddagger}$ J. Little,${ }^{\S}$ H. Özbay, ${ }^{\mathbb{Q}}$ and M. Samimy** \\ The Ohio State University, Columbus, Ohio 43210
}

\begin{abstract}
A study is presented of the modeling and implementation of different concepts for linear feedback control of a single-mode resonance shallow cavity flow. When a physics-based linear model is used for cavity pressure oscillations, an $\boldsymbol{H}^{\infty}$ controller was designed and tested experimentally. It significantly reduced the main Rossiter mode for which it was designed, while leading to strong oscillations at other Rossiter modes. Other linear control methods such as Smith predictor controller and proportional integral derivative (PID) controller exhibited similar results. The ineffectiveness of using fixed linear models in the design of controllers for the cavity flows is discussed. A modification of the PID design produced a parallel-proportional with time-delay controller that remedied this problem by placing zeros at the frequencies corresponding to other resonance states. Interestingly, it was observed that introducing the same zero to the $\boldsymbol{H}^{\infty}$ controller can also successfully avoid the strong oscillations at other Rossiter modes otherwise observed in the single-mode-based design. The parallel-proportional with time-delay controller was compared to a very effective open-loop method for reducing cavity resonance and exhibited superior robustness with respect to departure of the Mach number from the design conditions. An interpretation is presented for the physical mechanisms by which the open-loop forcing and the parallel-proportional with time-delay controllers reduce the cavity flow noise. The results support the idea that both controls induce in the system a rapid switching between modes competing for the available energy that can be extracted from the mean flow.
\end{abstract}

\section{Introduction}

$\mathbf{I}^{\mathrm{T}}$ $\mathrm{T}$ is widely recognized that the main reason to use feedback (closed-loop) control, as opposed to open-loop control, is to cope with the uncertainty, that is, to stabilize and improve the performance of a physical system under various operating conditions and modeling errors. ${ }^{1}$ Recently, several attempts have been made to apply closed-loop control methods to the control of flow phenomena. ${ }^{2-13}$ The current work is part of a multidisciplinary effort to develop tools and methodologies for closed-loop aerodynamic flow control. Initially this is done by choosing as a benchmark problem the control of the acoustic resonance of a flow over a shallow cavity. ${ }^{4-6}$ Although flow-induced cavity resonance is a well studied problem, the effects of the closed-loop dynamic control on the flow dynamics are not yet well understood. A recent paper by Cattafesta et al. ${ }^{3}$ provides a good review of the subject. Therefore, feedback controller design for closed-loop cavity flow control is still an open challenge. The

Received 30 November 2004; revision received 21 October 2005; accepted for publication 3 November 2005. Copyright (C) 2005 by M. Samimy. Published by the American Institute of Aeronautics and Astronautics, Inc., with permission. Copies of this paper may be made for personal or internal use, on condition that the copier pay the $\$ 10.00$ per-copy fee to the Copyright Clearance Center, Inc., 222 Rosewood Drive, Danvers, MA 01923; include the code 0001-1452/06 \$10.00 in correspondence with the CCC.

*Postdoctoral Researcher, Department of Electrical and Computer Engineering, Collaborative Center of Control Science; currently Senior Servo Engineer, Enterprise Servo Engineering, Seagate Technology, 1280 Disc Drive, Shakopee, MN 55379. Member AIAA.

${ }^{\dagger}$ Postdoctoral Researcher, Department of Mechanical Engineering, Gas Dynamics and Turbulence Laboratory, Collaborative Center of Control Science. Member AIAA.

${ }^{\ddagger}$ Graduate Student, Department of Electrical and Computer Engineering, Collaborative Center of Control Science.

${ }^{\S}$ Graduate Student, Department of Mechanical Engineering, Gas Dynamics and Turbulence Laboratory, Collaborative Center of Control Science. Student Member AIAA.

"I Professor, Department of Electrical and Computer Engineering, Collaborative Center of Control Science; currently Professor, Department of Electrical and Electronics Engineering, Bilkent University, Ankara 06800, Turkey.

** Professor, Department of Mechanical Engineering, Gas Dynamics and Turbulence Laboratory, Collaborative Center of Control Science; samimy.1@osu.edu. Associate Fellow AIAA. difficulty in the controller design lies in that the flow dynamics are governed by the Navier-Stokes equations, which, in control terminology, are infinite dimensional and highly nonlinear. These equations cannot be solved sufficiently fast for any practical models, and they cannot be used in any internal model control scheme.

Recently, a physics-based linear model was proposed and used in control of cavity oscillations. ${ }^{12,13}$ In the present work, we show that the parameters of this linear model can be optimized to match the open-loop response of the cavity pressure fluctuations at a given Mach number in a single-mode resonance case. Correspondingly, a robust feedback controller can be derived based on the $H^{\infty}$ mixed sensitivity minimization. The experimental results show that the $H^{\infty}$ controller reduces the dominant tone for which it is designed but introduces tones at other Rossiter modes. Similar observations can be obtained for other linear control methods, for example, Smith predictor control and proportional integral derivative (PID) control. In general, the experimental results do not match the expected results from the linear feedback theory, whereas the open-loop (in the absence of external forcing) system response of the linear model to a white noise fits the experimental data very well. Thus, we conclude that fixed linear time-invariant models, which do not take into account all possible Rossiter modes, are ineffective for controlling cavity flow resonance.

The real-time control implementations show that a significant improvement can be obtained by adding a tunable time delay between two proportional feedback loops, thus, placing a zero in the neighborhood of the additional resonant frequencies. The resulting parallel-proportional with time-delay controller is able to reduce the resonant tone without exciting additional tones. Compared to an equally effective open-loop system, ${ }^{14}$ it requires less control effort and exhibits better robustness with respect to departure of the Mach number from the design conditions. A physical interpretation of the effect of both controllers on reducing the cavity flow resonance is then explored.

A brief description of a linear model of the cavity flow based on the physics of the system is given in Sec. II. The $H^{\infty}$ controller design is also given in the same section. In Sec. III, the experimental apparatus and procedures used in this study and the characteristics of cavity flow resonance are described. The real-time implementations of the linear control designs and a comparison of openloop and closed-loop control techniques can be found in Sec. IV. Finally, in Sec. V, a physical interpretation of the mechanisms 
by which effective controllers reduce the cavity flow resonance is presented.

\section{Physics-Based Linear Model of the Cavity Flow and $\boldsymbol{H}^{\infty}$ Controller Design}

The physics-based linear model introduced in Williams et al. ${ }^{12}$ and Rowley et al. ${ }^{13}$ involves separate linear transfer function blocks for the shear layer $G(s)$, scattering $K_{S}>0$, acoustic feedback, and receptivity $K_{R}>0$, as shown in Fig. 1. The plant transfer function contains two internal feedbacks: acoustic feedback and receptivity. The shear layer can be taken to be a second-order system with a time delay:

$$
G(s)=\frac{\omega_{0}^{2} e^{-s \tau_{s}}}{s^{2}+2 \zeta \omega_{0} s+\omega_{0}^{2}}=G_{0}(s) e^{-s \tau_{s}}
$$

where $\omega_{0}>0,1>\zeta>0$, and $\tau_{s}>0$ are the parameters to be determined from the experimental data. If we define the acoustic feedback as

$$
\begin{gathered}
A(s)=e^{-s \tau_{a}} /\left[1-r(s) e^{-2 s \tau_{a}}\right], \quad \tau_{a}>0 \\
r(s)=r /\left(1+s / \omega_{r}\right)
\end{gathered}
$$

where $r>0$ and $\omega_{r}>0$, and model the receptivity feedback and scattering as constant gains, then the plant transfer function becomes

$$
P(s)=\frac{K_{S} G(s) A(s)}{1-K_{R} K_{S} G(s) A(s)}
$$

For more discussion on the physical meaning of the given parameters and possible ways to determine them, see Rowley et al. ${ }^{13}$ In this study, the parameters are optimized to match the open-loop response of single-mode resonance cavity pressure fluctuations at a given fixed Mach number. That is, when the feedback controller is taken out of the loop, the output power spectrum matches the power spectrum of the pressure fluctuations measured at the cavity floor center. Figure 2 shows this observation for the experimental data collected at Mach 0.3. The parameters of the plant that generate the response shown in Fig. 2 are listed in Table 1, where unity gains $K_{v}$ and $K_{m}$ are for the actuator and sensor dynamics. ${ }^{15}$ For these specific parameter values, the plant is stable. Note again that similar parameter tuning can be done at different Mach numbers, so that the open-loop response of the linear system shown in Fig. 1 matches the pressure fluctuations measured in the experiment (described in the next section). Also note that small changes in the Mach number may lead to relatively large changes in the optimized parameters (especially when Mach number variations result in changes in the

Table 1 Parameters of the plant shown in Fig. 1 to generate the results in Fig. 2

\begin{tabular}{lc}
\hline \hline Parameter & Value \\
\hline$\omega_{0}$ & $18,000 \mathrm{rad} / \mathrm{s}$ \\
$\zeta$ & 0.03 \\
$\tau_{s}$ & $0.0006 \mathrm{~s}$ \\
$\tau_{a}$ & $0.0001 \mathrm{~s}$ \\
$r$ & 0.01 \\
$\omega_{r}$ & $20,000 \mathrm{rad} / \mathrm{s}$ \\
$K_{R}$ & 0.01 \\
$K_{S}$ & 5.0 \\
$K_{v}$ & 1 \\
$K_{m}$ & 1 \\
\hline \hline
\end{tabular}

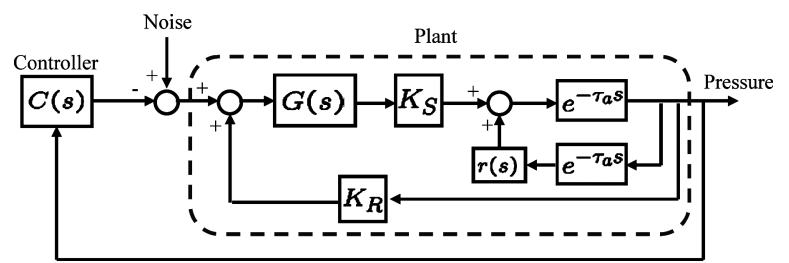

Fig. 1 Physics-based linear feedback system.

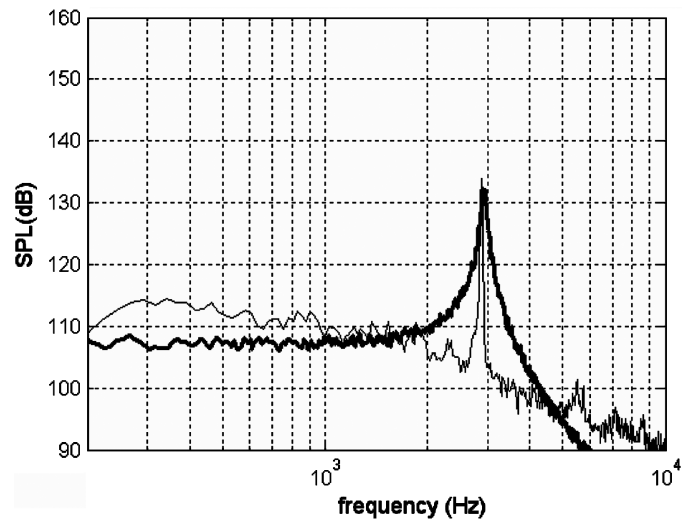

Fig. 2 Comparison of output power spectra: - , SPL spectrum from experimental data and $\longrightarrow$, SPL spectrum predicted by linear model.

number of dominant modes, as will be shown later). Therefore, it may be difficult to do gain scheduling for this set of models when flow conditions are varying. As a result, in the remaining parts of this section, for controller design purposes, we will assume that the Mach number is fixed.

The $H^{\infty}$ controllers can be designed ${ }^{1}$ to reduce the effect of the external noise on the system response. For cavity flow control, we can define a sensitivity minimization problem to compare the openloop and closed-loop responses of the system shown in Fig. 1. The open-loop system response is

$$
|P(j \omega)|
$$

and the closed-loop response is

$$
\frac{|P(j \omega)|}{|1+P(j \omega) C(j \omega)|}
$$

and so we would like to minimize the weighted sensitivity over all stabilizing controllers, the weight being the plant itself. Note that the function $(1+P C)^{-1}$ is the sensitivity function of the feedback system shown in Fig. 1. Similar techniques from linear robust control theory have been used for cavity flow control by Rowley and Williams. ${ }^{16}$ They have discussed the effects of actuator saturation as well, using describing function analysis.

To illustrate the $H^{\infty}$ design procedure, we consider the plant whose parameters take the numerical values given in Table 1 . The plant is stable and can be written in the form

$$
\begin{gathered}
P(s)=N_{o 1}(s) N_{o 2}(s) M_{n}(s) \\
N_{o 2}(s)=K_{S} G_{0}(s)=\frac{K_{S}}{\left(1+2 \zeta s / \omega_{0}+s^{2} / \omega_{0}^{2}\right)} \\
M_{n}(s)=e^{-h_{1} s}, \quad h_{1}=\tau_{s}+\tau_{a} \\
N_{o 1}(s)=\left[1-K_{R} N_{o 2}(s) M_{n}(s)-r(s) M_{2}(s)\right]^{-1} \\
M_{2}(s)=e^{-2 \tau_{a} s}
\end{gathered}
$$

Because the plant and the weight are infinite dimensional, there is no direct and easy solution to this weighted sensitivity minimization problem. For the case where the plant, or the weight, is finite dimensional, the problem can be solved using certain procedures from operator theory. ${ }^{17}$ In this particular case, we approximate the weight by another infinite-dimensional transfer function that captures the essential dynamics within a large-frequency region. Then, by exploiting this special structure, we solved the problem as detailed by Yuan et al. ${ }^{15}$ The controller is in the form

$$
C(s)=C_{2}(s)\left(1-r(s) M_{2}(s)\right)+K_{R}
$$

where

$$
C_{2}(s)=\left(\frac{\gamma}{\gamma_{\min }}-\frac{\gamma_{\min }}{\gamma}\right) \frac{N_{o 2}(s)^{-1}}{\left(1+a s+b s^{2}\right)}\left(\frac{1}{1+H(s)}\right)
$$

$$
H(s)=H_{\mathrm{FIR}}(s)+H_{\mathrm{IIR}}(s)
$$


These finite impulse response (FIR) and infinite impulse response (IIR) filters, as well as all of the other parameters (including $\gamma$, $\gamma_{\min }, a$, and $b$ ) appearing in the preceding formula, can be explicitly computed. ${ }^{15}$ Note that the controller is infinite dimensional, but it can be implemented using finite-dimensional terms, delay blocks, and an FIR filter. In fact, the controller was implemented easily within the hardware restrictions (digital signal processing and input/output capabilities) imposed by the dSPACE real-time control system we used. See Sec. III for the detailed description of the experimental setup.

The numerical simulation result shown in Fig. 3 shows that when the plant is taken to be the linear model as already defined, then the $H^{\infty}$ controller is able to suppress the strong sinusoidal oscillations seen at the output. Implementations of this type of controllers are discussed in Sec. IV.

\section{Experimental Apparatus and Analysis}

In this section we briefly outline the experimental setup that is described in more detail by Debiasi and Samimy. ${ }^{14}$ It consists of an optically accessible, blowdown-type wind tunnel with a test section of width $W$ and height $H$ equal to $50.8 \mathrm{~mm}$. A cavity that spans the entire width of the test section is recessed in the floor with a depth $D=12.7 \mathrm{~mm}$ and length $L=50.8 \mathrm{~mm}$ for an aspect ratio $L / D=4$. The cavity shear layer is forced by a two-dimensional synthetic-jettype actuator issuing from a slot of height $h=1 \mathrm{~mm}$ embedded in the cavity leading edge (Fig. 4). The angle of the actuator flow to the main flow is $30 \mathrm{deg}$. This assembly has a mean actuator to main flow momentum ratio $C_{\mu}=h u^{2} / H U_{\infty}^{2}$ in the range $10^{-4}-10^{-6}$, where $u$

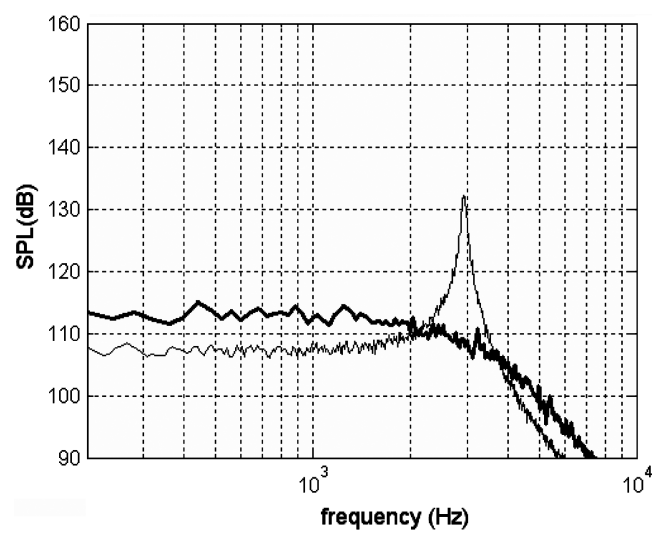

Fig. 3 Prediction of $\boldsymbol{H}^{\infty}$ controller performance for plant described by linear model (linear simulation result): - SPL spectrum without control, and -, SPL spectrum with control.

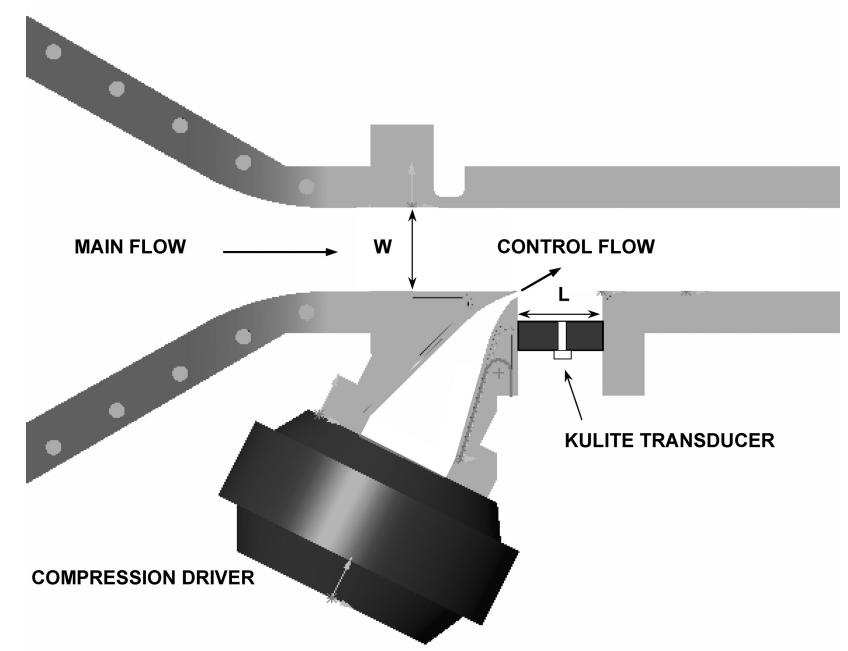

Fig. 4 Facility cutout: converging nozzle, test section, cavity, actuator coupling, and position of Kulite transducer in cavity floor. is the rms value of forcing velocity at the actuator exit slot and $U_{\infty}$ is the velocity of the freestream in the test section above the cavity.

Pressure fluctuations were measured by Kulite dynamic pressure transducers placed in different locations in the test section and at the actuator exit. The Kulite signals were bandpass filtered between 200 and $20,000 \mathrm{~Hz}$ to remove spurious frequency components. A dSPACE 1103 controller board connected to a Dell Precision Workstation 650 computer was used to acquire at $50 \mathrm{kHz}$ through a 12-bit channel the signal from a transducer flush-mounted in the middle of the cavity floor and to manipulate it to produce the desired control signal in a 14-bit output channel. To maximize the control board performance, its processor was used exclusively for running the control routines. Simultaneous recordings of 32 blocks of 8192 points (262,144 samples) were acquired at $200 \mathrm{kHz}$ through a 16-bit resolution acquisition board (National Instruments PCI-6036E) operating independently in the computer. With the Kulite sensitivity and the amplifier gain setting accounted for, the voltage values of the timetraces were converted to nondimensional pressure referenced to the commonly used value of $20 \mu \mathrm{Pa}$. Short-time Fourier transform (STFT) was used to provide information on the time evolution of the frequency content of the unsteady pressure signals. The STFT of a signal $x(t)$ is defined as ${ }^{18,19}$

$$
\operatorname{STFT}(t, f)=\int_{-\infty}^{\infty} x(\tau) w(\tau-t) e^{-j 2 \pi f \tau} \mathrm{d} \tau
$$

where $w(t)$ is the window function (in the present analysis the Hanning window). The STFT power spectra were computed by splitting the signal into segments with about $95 \%$ overlap, windowing each with the window function, calculating a 8192-point fast Fourier transform (which provided a spectral resolution of about $24 \mathrm{~Hz}$ ), and converting them to sound pressure level (SPL) values. An important consequence of the uncertainty principle is that the time-bandwidth product is never less then $1 / 2$ :

$$
\Delta t \cdot \Delta \omega \geq 1 / 2
$$

Thus, a compromise is required between time and frequency resolution. The values of sampling frequency and of the spectral resolution utilized in the present work guarantee a time resolution $\Delta t$ of no less that $3.3 \mathrm{~ms}$. The corresponding spectrograms are visualized as contour plots and provide a representation of the time-dependent SPL values of the time series. Time averaging of the SPL values obtained as described produced the SPL spectra (accurate within $\pm 1 \mathrm{~dB}$ ) presented in this study.

The instantaneous features of the flow in a streamwise plane at the test section centerline were visualized by the scattering of a laser light sheet from a Continuum Nd:YAG pulsed laser operating at a wavelength of $532 \mathrm{~nm}$ and entering the test section from an optical window on the top wall of the tunnel. Locally seeded smoke entering the cavity from a streamwise slot at the floor of the cavity was used for flow visualizations. Images were acquired using a Roper Scientific intensified charge-coupled device camera. The camera and the laser were synchronized to a reference acoustic signal from the flow for acquisition of phase-locked images. For every flow condition explored, eight sets of phase-locked images were obtained, each corresponding to an incremental phase shift of one-eighth of the resonant or forced period. Ensemble-average images were obtained from the individual images of each set.

Debiasi and Samimy ${ }^{14}$ observed that the experimental facility exhibits strong, single-mode resonance in the Mach number ranges $0.25-0.31$ and $0.39-0.5$ and multimode resonance in the Mach number range 0.32-0.38 as shown in Fig. 5, where dominant peaks are represented by closed circles and open circles represent other peaks that appear during multimode resonance. Shown also are the frequencies predicted by the semi-empirical formula of $\operatorname{Rossiter}^{20}$ (also see Ref. 21) and the cavity first longitudinal and transversal (vertical) modes. In the same study, Debiasi and Samimy ${ }^{14}$ observed that the frequency of sinusoidal forcing with the synthetic-jet-type actuator has a major impact on the cavity flow resonance, whereas the effect of the amplitude is relatively minor and affects the control 


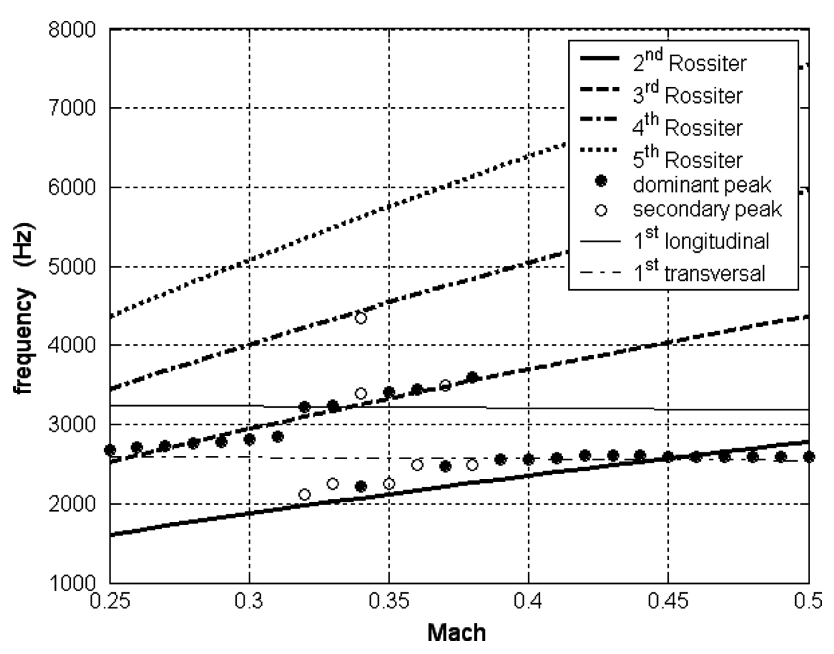

Fig. 5 Frequencies function of flow Mach number: - Rossiter; $\ldots$, cavity first longitudinal and transversal; and $\bigcirc$, measured.

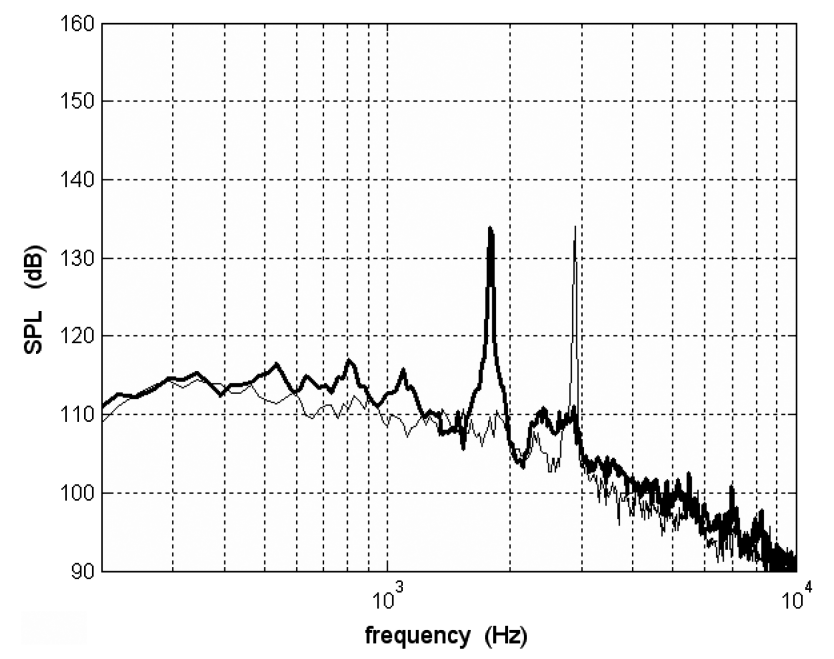

Fig. 6 Effect of $H^{\infty}$ control on Mach 0.30 flow: - , SPL spectrum of uncontrolled flow and - , SPL spectrum of controlled flow.

authority only at higher Mach numbers. This prompted the development of a logic-based type of control that searches, in a closed-loop fashion, the forcing frequencies that reduce the cavity flow resonant peaks and then maintains the system in such conditions through an open-loop, optimal frequency forcing (OPFF). The technique performed well in the experimental trials and allowed identification of optimal frequencies for the reduction of resonant peaks in the explored Mach number range 0.25-0.5.

\section{Experimental Results}

\section{A. Linear Control Implementations on the Experimental Setup}

We explore real-time implementations of linear feedback controllers in Simulink/dSPACE systems. The $H^{\infty}$ controller was designed for Mach 0.3 , a flow dominated by strong single-mode resonance at about $2800 \mathrm{~Hz}$ (thin line in Fig. 6). The controller diagram for the real-time implementations is shown in Fig. 7. It suppressed the main frequency of oscillation (third Rossiter mode), but it led to strong oscillations with frequency about $1900 \mathrm{~Hz}$ (Fig. 3), which corresponds to the second Rossiter mode for this flow (Fig. 5). Similar results were obtained for other types of linear feedback controllers, for example, Smith predictor and PID controller. We refer to Yan et al. ${ }^{22}$ for details. Such behavior has been observed by other researchers using linear feedback control. ${ }^{12,23,24}$

In the case of the PID controller, we noticed that the integral term did not have any significant effect on the system response due to the nature of the actuator. By replacing the derivative component with a first-order filter with an adjustable cutoff frequency $1 / \tau_{d}$, we obtained a PD-like controller in the form

$$
C_{\mathrm{PD}}(s)=K_{p}+K_{d}\left[\tau_{d} s /\left(\tau_{d} s+1\right)\right]
$$

Optimal parameters for elimination of the main frequency of oscillation (third Rossiter mode) of the Mach 0.30 flow were found from a brute force search method. They turn out to be $K_{p}=8, K_{d}=0.04$, and $\tau_{d}=200$, but these parameters led to strong oscillations in the neighborhood of the second Rossiter mode, as shown in Fig. 8. The signals $\mathrm{P}, \mathrm{D}$, and the control spectra correspond to voltage signals produced by the controller and not to pressure fluctuation levels. Nevertheless, for the sake of comparison, they are presented alongside the SPL spectra from the Kulite transducers placed at the actuator exit and in the middle of the cavity floor. Therefore, the decibel levels in Fig. 8 refer only to the spectra of the Kulite signals.

These experimental observations confirm that the plant is nonlinear and linear designs lead to results that cannot be predicted from the linear analysis. Meanwhile, the linear controllers were designed for the unforced, single-mode resonant flow, and thus, the potential excitement of the other Rossiter modes was not taken into account. We could address this issue by selecting the uncertainty weight of the $H^{\infty}$ control to include sharp peaks at these Rossiter modes (which do not appear in the open-loop response). However, this will further complicate the $H^{\infty}$ design, which is already complicated due to infinite dimensionality of the plant and weights. We found a simple solution in the form of a parallel-proportional control with time delay and, thus, concentrate on this simple controller in the rest of the paper. Observations of the control signals in Fig. 8 motivated us to add a tunable time delay $h$ to the second term in Eq. (12), which introduced a phase shift for signals operating in the neighborhood of the second Rossiter frequency,

$$
C_{\mathrm{PD}}(s)=K_{p}+K_{d}\left[\tau_{d} s /\left(\tau_{d} s+1\right)\right] e^{-h s}
$$

Furthermore, having observed that, due to the relatively large value of $\tau_{d}$, this controller acts like a parallel-proportional (PP) control with individual delay terms, we simplified the controller as

$$
C_{\mathrm{PP}}(s)=K_{p}\left(1+e^{-h s}\right)
$$

A schematic of such a controller as implemented in our experimental setup is given in Fig. 9. We verified that the performance of this controller is very much like the performance of the PD-like controller with the same time delay.

A value of $h=260 \mu$ s was used to introduce a 180-deg phase shift for signals operating in the neighborhood of the second Rossiter mode, thus, effectively placing a zero at the corresponding frequency. In fact, it is straightforward that

$$
1+\exp \left(-2.6 \times 10^{-4} 2 \pi 1932 j\right)=0
$$

As a result, the performance of this controller is greatly improved, as shown in Fig. 10a where, similar to Fig. 8, one can observe the spectra of the signals in different points of the closed loop. In Fig. 10, both controllers have $260-\mu$ s time delay between control channels. The control signals were processed as the pressure transducers signals. Reference decibel levels are provided only for the SPL spectra of the pressure transducer signals. Combining the $260-\mu$ s phase-shifted $\mathrm{P}$ signals produces the control signal whose spectrum is characterized by frequency cancellation at $1932 \mathrm{~Hz}$ and its odd harmonics, whereas a modest reinforcement is produced at the even harmonics. Note that the highest peak of the forcing signal at the actuator exit occurs at a frequency of about $3800 \mathrm{~Hz}$, a value close to $3920 \mathrm{~Hz}$, one of the optimal forcing frequencies for reducing the resonance of the Mach 0.30 cavity flow. ${ }^{14}$

In a similar fashion, we also introduced the same zero of the PP control in the $H^{\infty}$ controller

$$
C_{h \text { inf }}(s)=C(s)\left(1+e^{-h s}\right)
$$

where $C(s)$ defined in Eq. (7) is the $H^{\infty}$ controller designed for single-mode resonance. As shown in Fig. 10b, this revised controller can also successfully avoid oscillations at the second Rossiter 


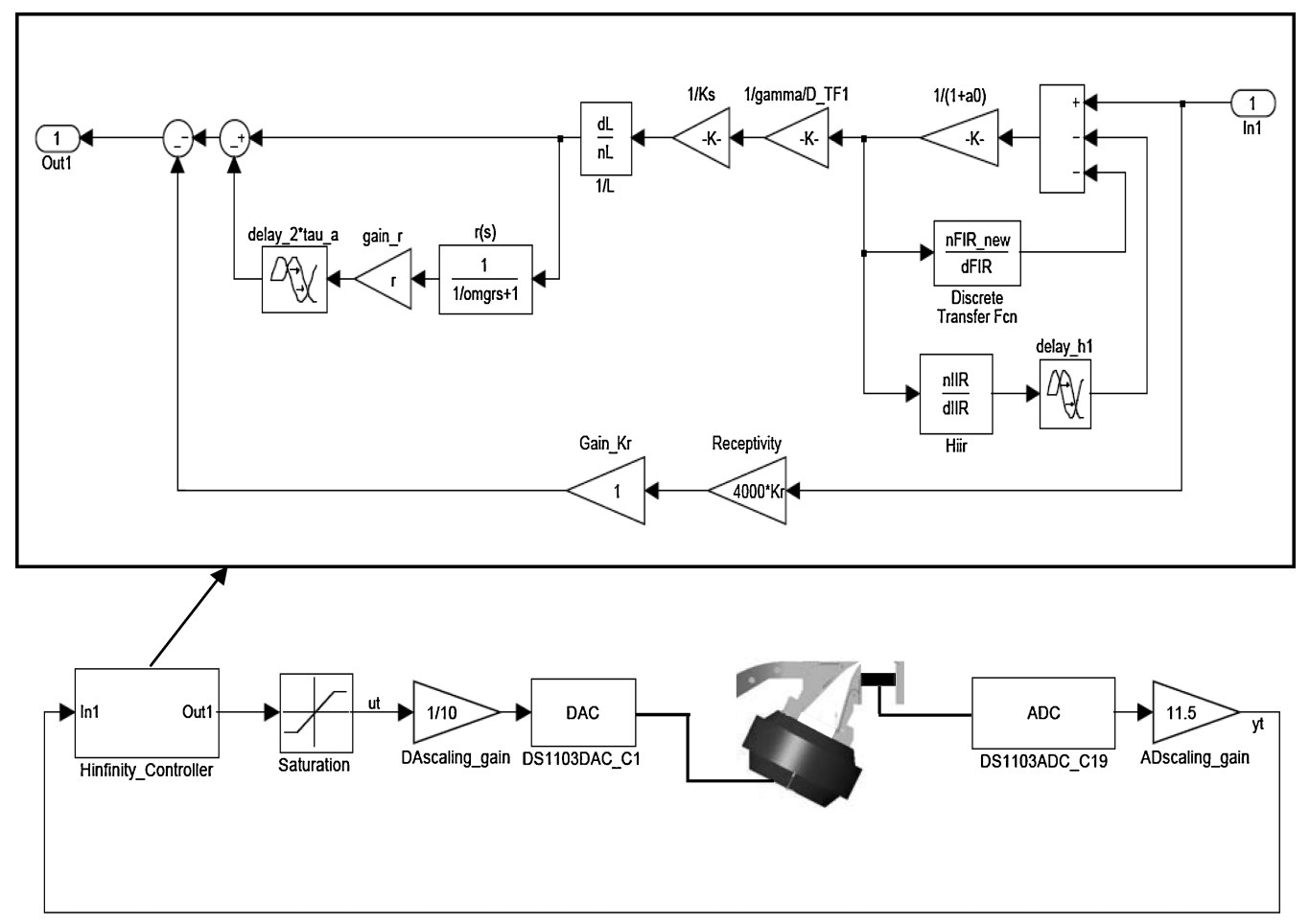

Fig. 7 Diagram of $H^{\infty}$ controller.

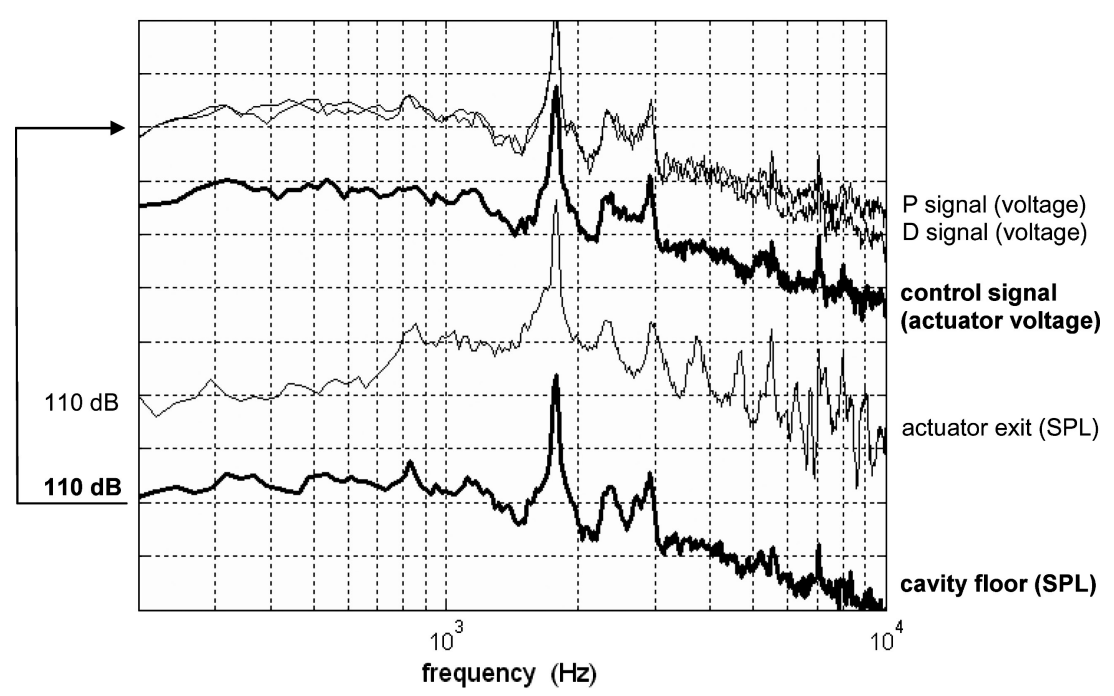

Fig. 8 Spectra of Mach 0.30 cavity flow system excited by the PD-like (effectively a proportional) controller.

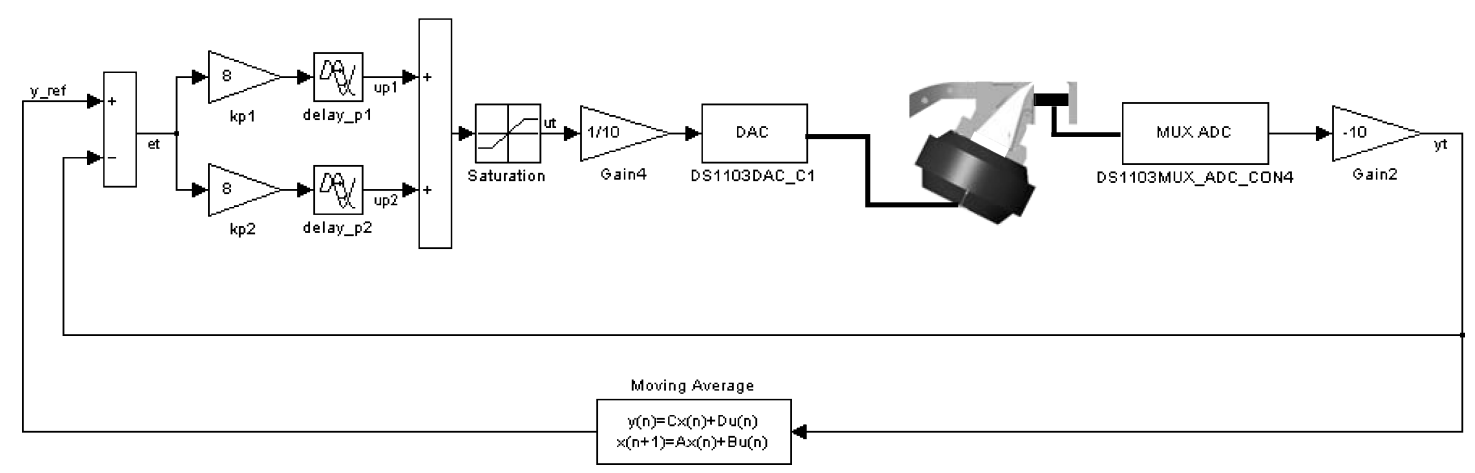

Fig. 9 Diagram of PP controller with time delay. 

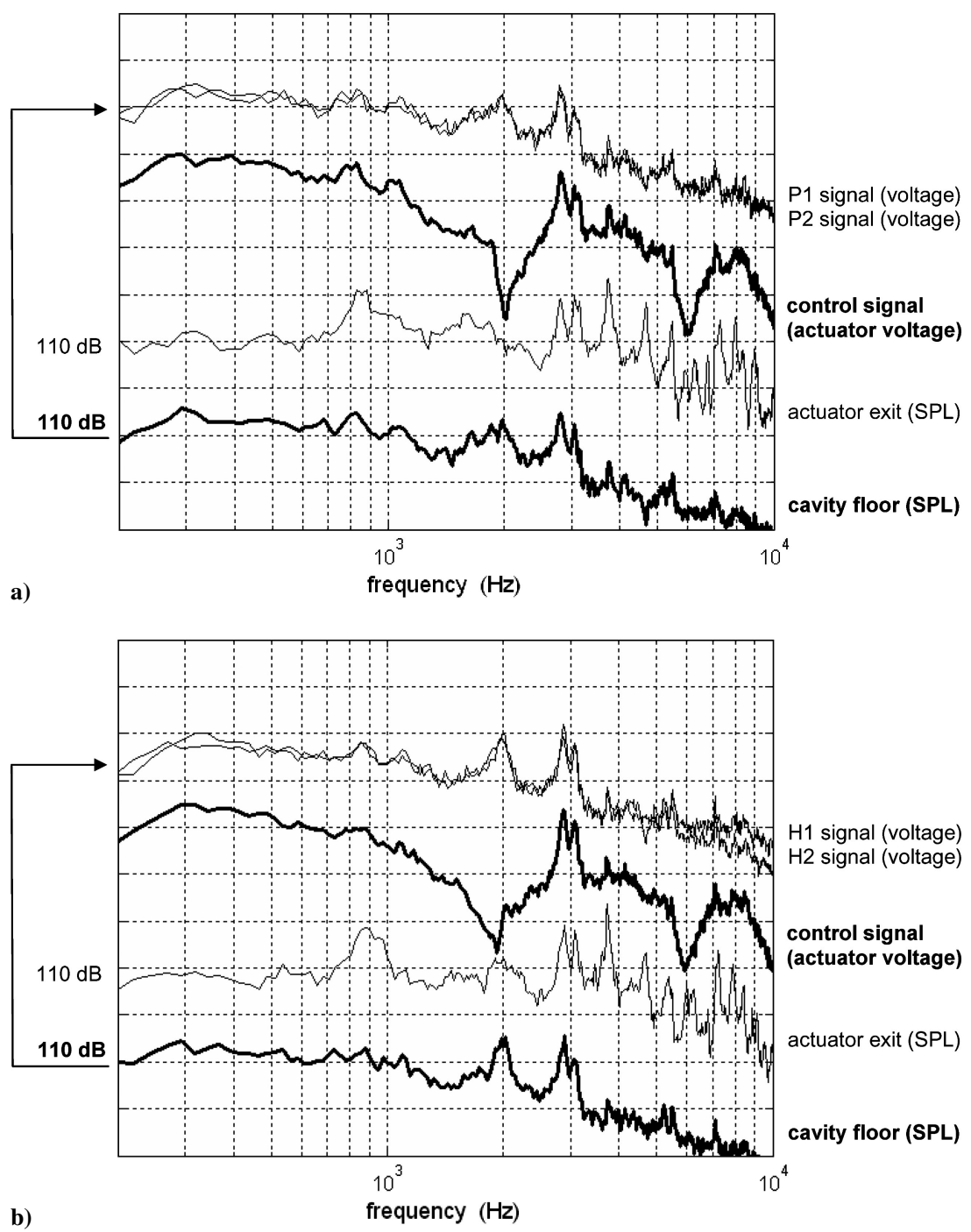

Fig. 10 Spectra of Mach 0.30 cavity flow system controlled with a) PP with time delay control and b) $H^{\infty}$ with time delay control.

mode. This observation further confirms that the ineffectiveness of using fixed linear models for controller design results from potential excitations of other Rossiter modes.

\section{B. Comparison of Optimal Forcing Frequency and PP Control}

In the preceding section we have illustrated how the simple PP controller with proper time delay between the two feedback loops is effective in reducing the cavity flow resonance at Mach 0.3. Analogous results, not shown here, were also obtained at other Mach numbers ${ }^{22}$ and provided peak noise reductions comparable with those obtained using the OPFF control discussed in more detail by Debiasi and Samimy. ${ }^{14}$

In this section, we compare the performance of these two methods by using them to suppress the resonance of flows at different Mach numbers and by assessing their robustness with respect to flow variations. For simplicity, we will not provide the comparison results on the $H^{\infty}$ controller with time delay [Eq. (17)], which exhibits similar closed-loop results to the PP with time-delay controller.

Figure 11 shows the effect of the OPFF control on flows close to Mach 0.3, a flow condition that has been used as a benchmark case in our studies. ${ }^{14}$ The OPFF forcing voltage was a sinusoidal wave at $3920 \mathrm{~Hz}, 2.5 \mathrm{~V}_{\mathrm{rms}}$, which corresponds to one of the optimal conditions for reducing the resonant peak of the Mach 0.30 flow without introducing other strong spectral peaks. In Figs. 11a-11c, the thin line is the SPL spectrum of the original unforced case, whereas the thick line corresponds to the spectrum with OPFF. Be- low Mach 0.30, the unforced flow exhibits a strong, single-mode resonant peak at about $2800 \mathrm{~Hz}$ (Fig. 5), whereas above Mach 0.31 , the resonance exhibits multimode behavior. OPFF forcing significantly reduces the resonant peak at Mach 0.29 (Fig. 11a), that is, a flow very close to the conditions for which OPFF was tuned. This occurs without introduction of any peak other than the forcing one, which, in any case, does not significantly exceed the other spectral components. This is not the case at Mach number slightly below or above these values. At Mach 0.27 (Fig. 11b), the original resonant tone is reduced, but the forcing one exceeds the other components by more than $9 \mathrm{~dB}$. At Mach 0.31 , a condition where the unforced flow is transitioning to multimode resonance (Fig. 11c), the forcing peak is strong and only marginally inferior to the strongest unforced tone. Additionally, forcing has increased the second Rossiter mode that is close to a subharmonic of the forcing tone.

From Fig. 11, it is clear that OPFF, although a simple and effective method that reduces the resonance at the Mach number for which it is tuned, is very sensitive to variations in the flow conditions. This behavior has been confirmed by repeating this experiment for flows in the neighborhood of other Mach numbers for which the resonance was suppressed with appropriate OPFF tuning.

Figure 12 presents the results of a similar trial using the PP with time-delay controller whose parameters are optimized for reducing the resonance of the Mach 0.30 flow. In Figs. 12a-12c, the thin lines are the same SPL spectra for the unforced cases as in Fig. 11. Application of the PP control to the Mach 0.29 flow (Fig. 12a) provides a 


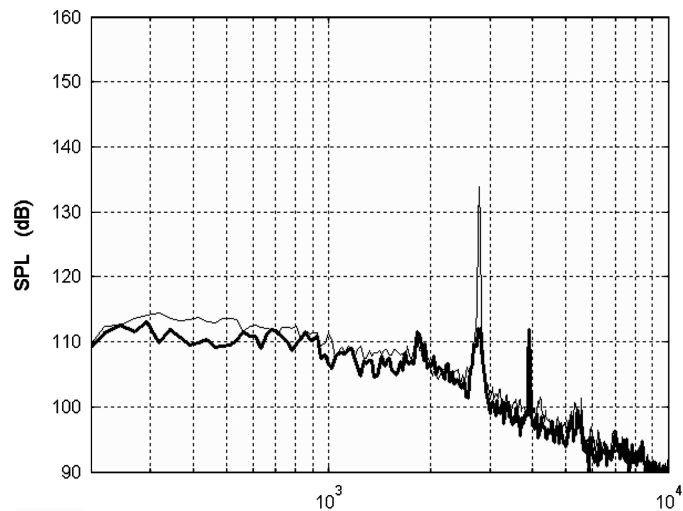

a)

frequency $(\mathrm{Hz})$

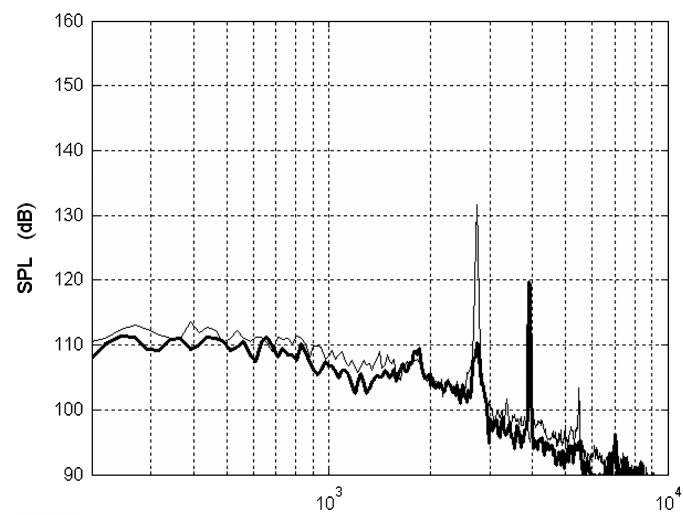

b)

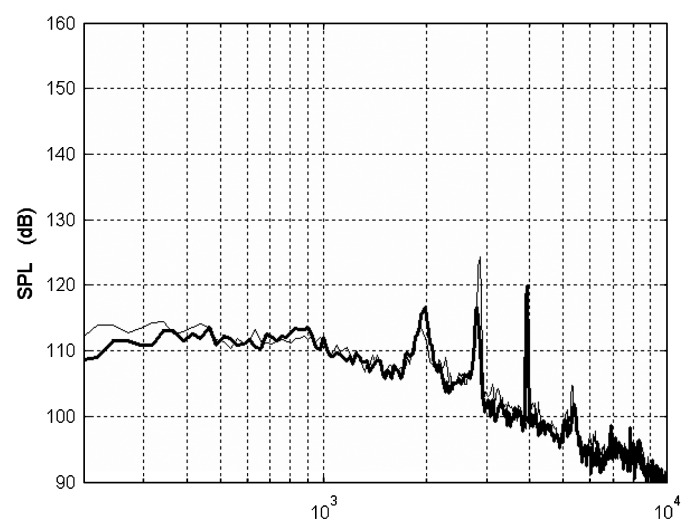

c)

frequency $(\mathrm{Hz})$

Fig. 11 Effect on different flows of OPFF control at $3920 \mathrm{~Hz}, 2.5 \mathrm{~V}_{\mathrm{rms}}$; , unforced flow SPL spectrum and - spectrum with OPFF control at a) Mach 0.29, b) Mach 0.27, and c) Mach 0.31.

reduction of the resonant peak similar to that of the OPFF control in Fig. 11a. The PP control appears to have spread the acoustic energy among the frequencies surrounding the Rossiter modes, and it has not introduced a strong actuation peak. Interestingly, and in contrast with what was observed in Fig. 11 for the OPFF case, the same general characteristics and benefits are maintained when the PP control is applied to the Mach 0.27 and Mach 0.31 flows (Figs. 12b and $12 \mathrm{c}$ ). In both cases, the maximum spectral peak is lower than that produced with OPFF and the PP control does not introduce a strong forcing tone. Additional measurements, not presented here, reveal that, whereas the performance of the PP controller remained equally good at Mach numbers lower than 0.27, it deteriorated at Mach numbers larger than 0.32 .

Comparison of Figs. 11 and 12 clearly indicates that, unlike the OPFF control, the PP with time-delay controller exhibits good robustness with respect to some departure from the design Mach number, that is, to variations that do not lead to big changes of the Rossiter frequencies, for example, second and third Rossiter modes. This is

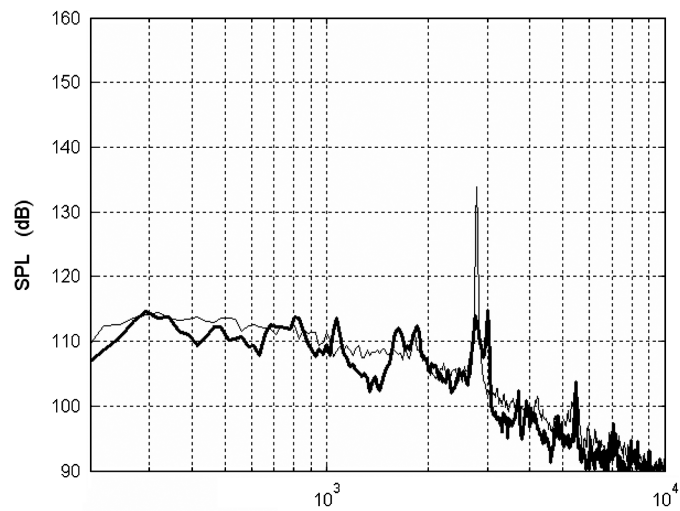

a)

frequency $(\mathrm{Hz})$

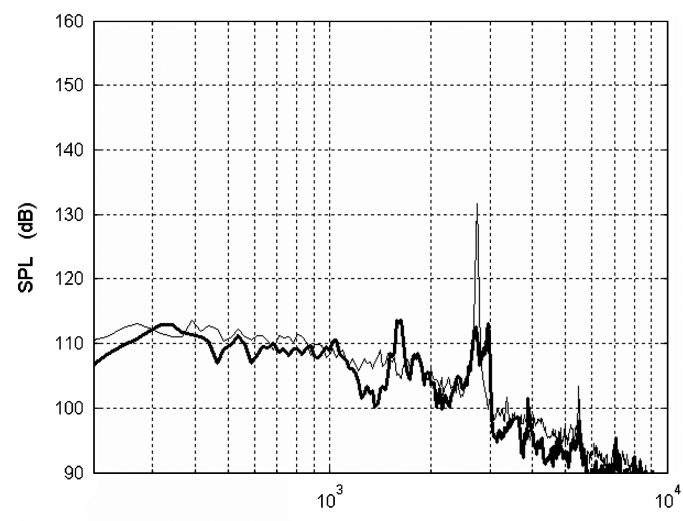

b)

frequency $(\mathrm{Hz})$

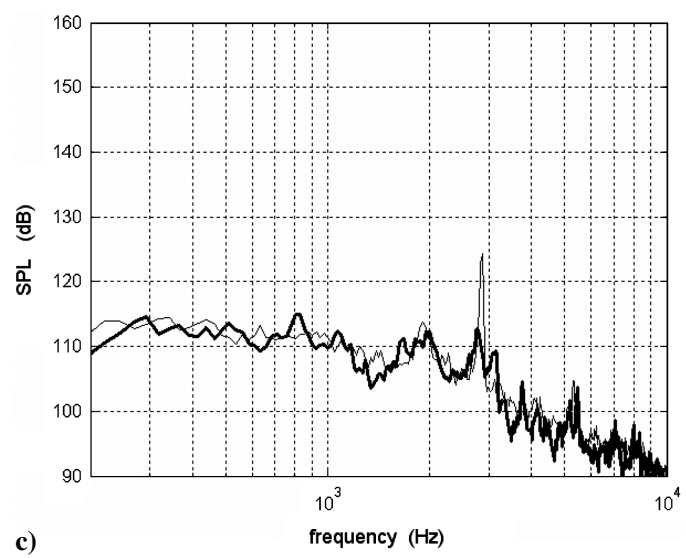

Fig. 12 Effect on different flows of PP control with time delay tuned for Mach 0.3 ; — with PP control at a) Mach 0.29, b) Mach 0.27, and c) Mach 0.31.

not surprising because OPFF is an open-loop method, where the optimal frequency is tuned with the assumption that the input is always a sinusoidal signal with given amplitude. In contrast, the PP method is a feedback control design that is more impervious to changes of the open-loop plant as long as these are relatively small. Finally, note that the results presented in Fig. 11 for the OPFF control were obtained with actuation at $2.5 \mathrm{~V}_{\text {rms }}$, whereas those in Fig. 12 for the PP controller were obtained operating the actuator at about $2 \mathrm{~V}_{\mathrm{rms}}$, that is, with less than two-thirds of the power required in the OPFF case (the power being proportional to the square of the rms voltage). These results compare well with Cattafesta et al ${ }^{25}$ findings on the adaptability and lower power consumption of the closed-loop control in comparison with open-loop control.

\section{Physical Interpretation}

In this section, we provide an interpretation of the physical mechanisms by which the OPFF and the PP with time-delay control techniques reduce the cavity-flow noise. Debiasi and Samimy ${ }^{14}$ 


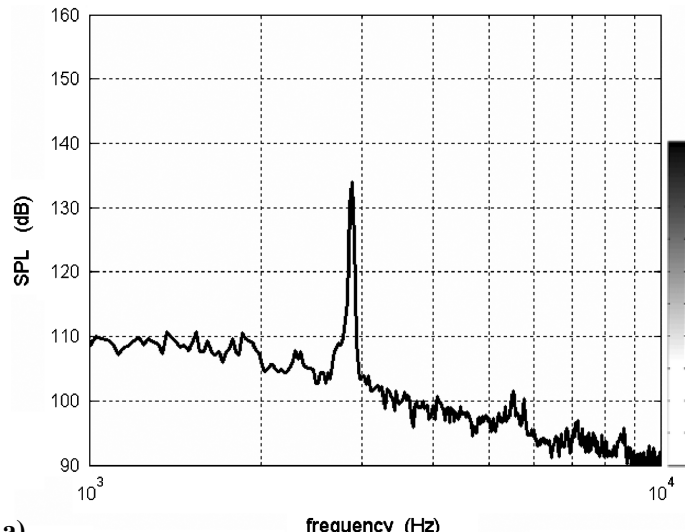

a)

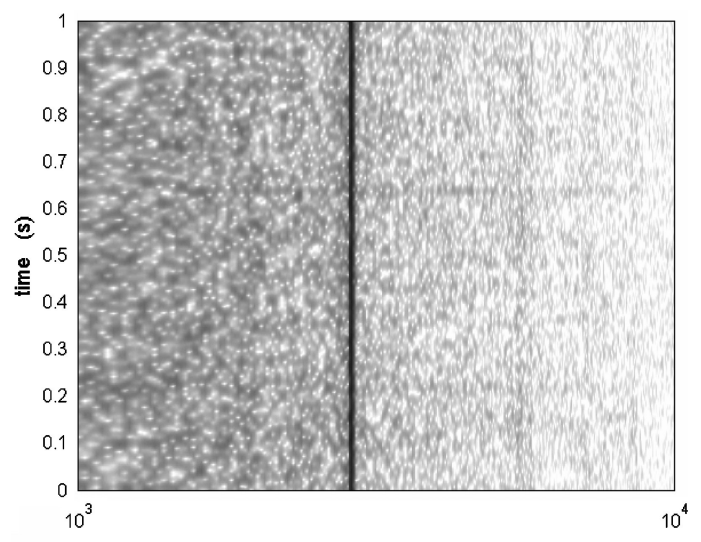

b)

frequency $(\mathrm{Hz})$

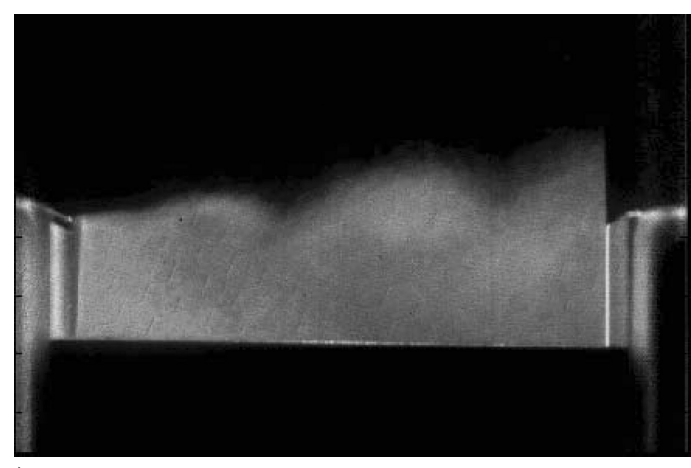

c)

Fig. 13 Single-mode resonating Mach 0.30 flow: a) SPL spectrum, b) spectrogram, and c) phase-locked average laser light-scattering image.

speculated that OPFF control induces in the system a state similar to multimode resonance where rapid switching occurs between the resonant and the forcing modes. Because this switching, although rapid, is not instantaneous, part of the available energy either remains in the mean flow or dissipates in the switching process, which produces a less efficient resonant system where no single mode locks in to dominate the spectrum. In commenting on similar peak reductions obtained with open-loop forcing, Cattafesta et al. ${ }^{25}$ suggested that a competition exists between the fundamental mode and the forced mode for the available energy that can be extracted from the mean flow. Here we want to verify this hypothesis more exactly and investigate if a similar or a different mechanism also applies to the case of PP control. To this end, we contrast the SPL spectra, spectrogram, and the average centerline laser light-scattering images of representative single-mode resonating, multimode resonating, and controlled flow cases.

Figure 13 presents the SPL spectrum (Fig. 13a) and the corresponding spectrogram (Fig. 13b) of the single-mode resonating Mach 0.30 flow in the range $1-10 \mathrm{kHz}$. For ease of comparison, the bar of the spectrogram intensity is placed next to the corresponding
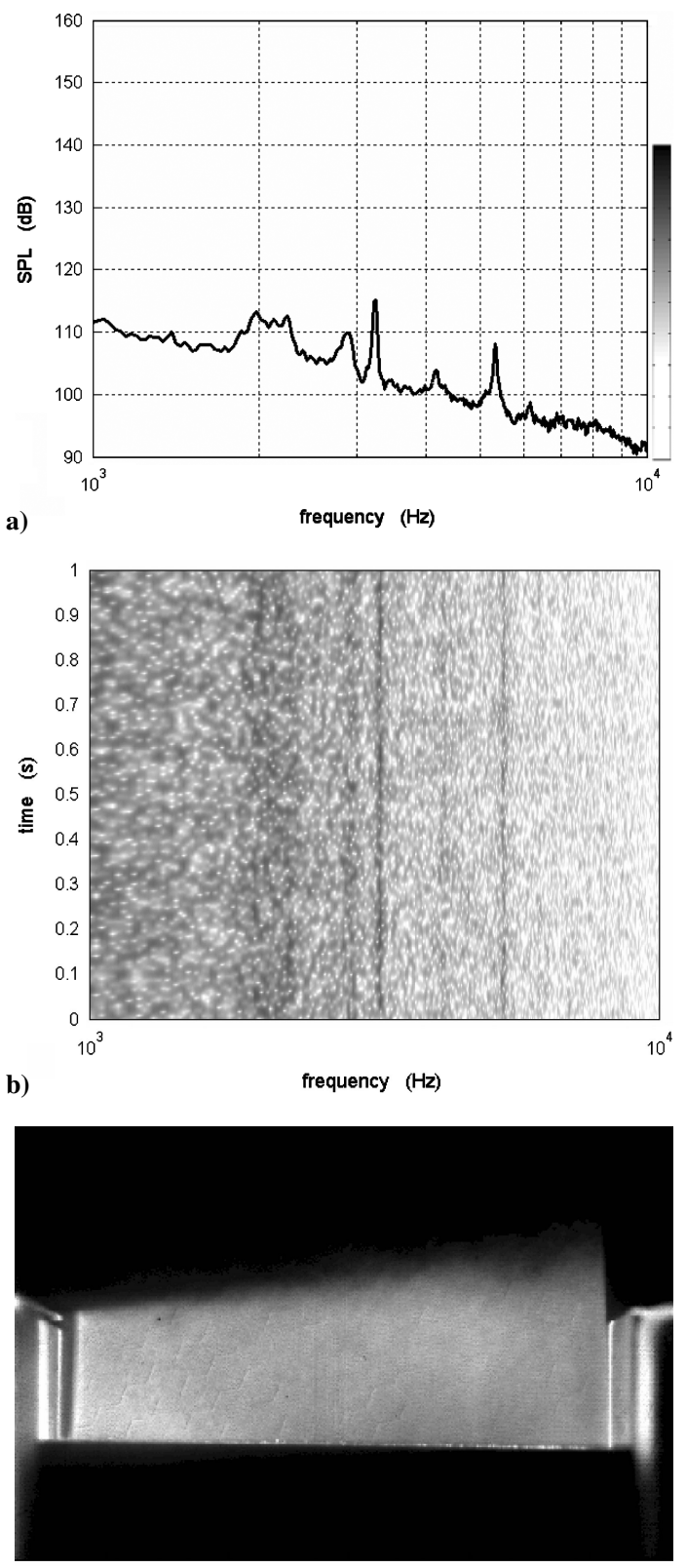

c)

Fig. 14 Multimode resonating Mach 0.32 flow: a) SPL spectrum, b) spectrogram, and c) phase-locked average laser light-scattering image.

spectrum values. The spectrogram confirms that, as expected, the strong resonant tone is time independent in contrast with the lower spectral components that change in time as evidenced by the spread of dots for frequencies below the resonant one. Figure $13 \mathrm{c}$ is an average visualization of the centerline flow obtained by phase locking the laser and the camera to the resonant peak. Both Fig. 13 and the instantaneous images from which it was obtained show very clearly the presence of three structures corresponding to the third Rossiter mode at which this flow resonates.

Figure 14 presents the SPL spectrum, spectrogram, and phaseaveraged centerline image of the multimode resonating Mach 0.32 flow. Clearly visible in the spectrum (Fig. 14a) are the peak corresponding to the longitudinal mode close to the third Rossiter mode at about $3250 \mathrm{~Hz}$ and a broader peak around the second Rossiter mode slightly above $2000 \mathrm{~Hz}$. Visible is a very small peak at the fourth Rossiter mode at about $4200 \mathrm{~Hz}$, the stronger fifth Rossiter mode at $5200 \mathrm{~Hz}$, and also a remnant at $2850 \mathrm{~Hz}$ of the third Rossiter mode for slower flow conditions (Fig. 5). The spectrogram (Fig. 14b) reveals the rapid switching between these modes as evidenced by the discontinuous time sequence of darker dots at these frequencies. Of 


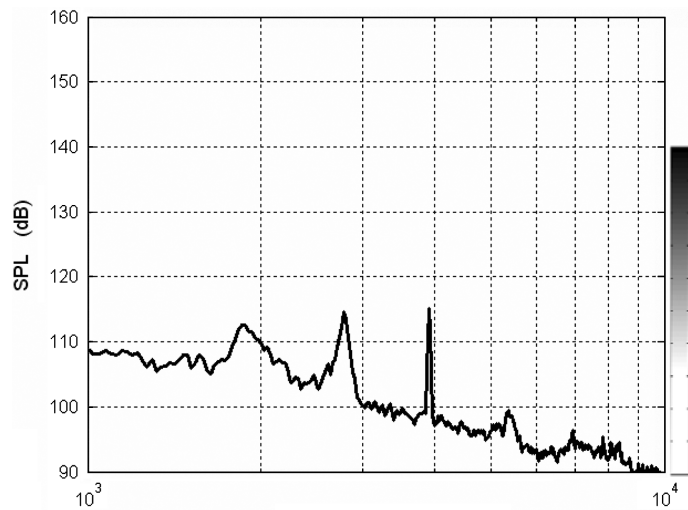

a)

frequency $(\mathrm{Hz})$

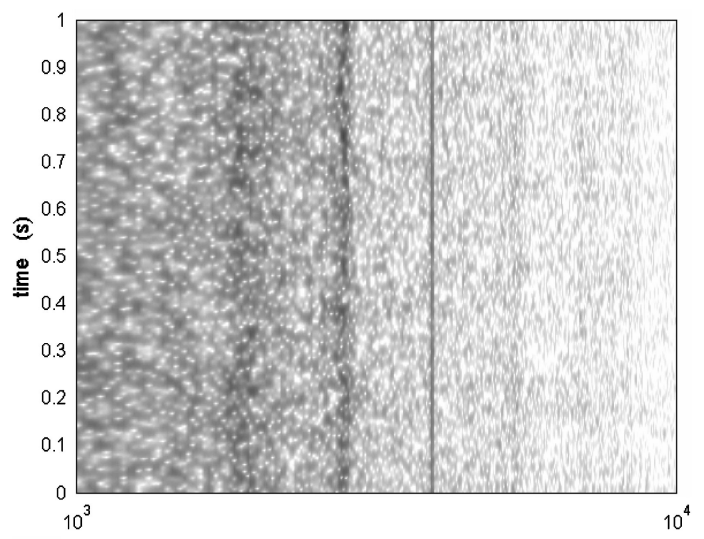

b)

frequency $(\mathrm{Hz})$

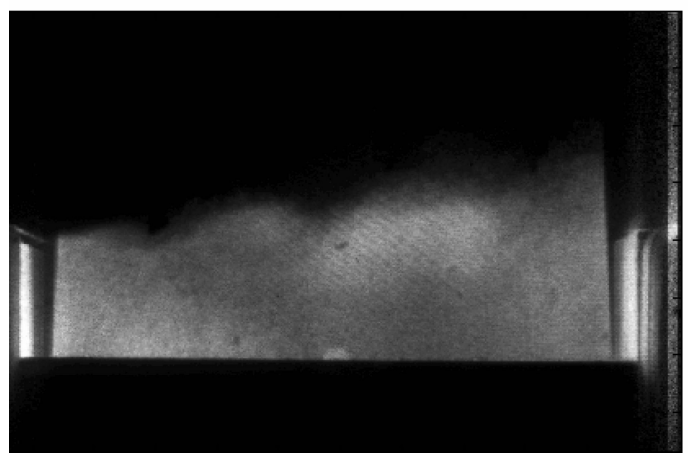

c)

Fig. 15 Mach 0.30 flow with OPFF control at $3920 \mathrm{~Hz}, 2.5 \mathrm{~V}_{\mathrm{rms}}$ : a) SPL spectrum, b) spectrogram, and c) phase-locked average laser lightscattering image.

particular interest is the unusually broad peak spanning the 1950$2300-\mathrm{Hz}$ region in the neighborhood of the second Rossiter mode. As a result, the acoustic energy of this flow is well spread across the spectrum and, save for the tones corresponding to the longitudinal and fifth Rossiter modes, it approaches the ideal behavior of a quieter, broadband flow. In contrast with the centerline image of the Mach 0.30 flow, the phase-averaged centerline image of the Mach 0.32 flow (Fig. 14c) does not reveal any significant shear layer feature. This is attributable to either the inability to lock the laser and camera to a steady peak or the lack of quasi periodicity of the structures in the shear layer or a combination of the two because structures were clearly observed in the instantaneous images.

Figure 15 presents the SPL spectrum, spectrogram, and average centerline image of the Mach 0.30 resonance suppressed with the OPFF at $3920 \mathrm{~Hz}, 2.5 \mathrm{~V}_{\text {rms }}$ control. This forcing frequency, which is time independent as evident in the spectrogram (Fig. 15b) is very close to the first harmonic of the second Rossiter mode of about $1900 \mathrm{~Hz}$ for this Mach number, as well as being close to the fourth
Rossiter mode at $4000 \mathrm{~Hz}$ (Fig. 5). As a result, the forcing tone slightly enhances the second Rossiter mode that, thus, competes with the third mode, establishing the mode switching between them clearly visible in the spectrogram. This supports the idea that OPFF induces a switching between resonant modes; these can be either the Rossiter modes or other natural modes of the cavity. Different from the preceding case, the average centerline image in this flow exhibits four structures when locking the laser and the camera to the steady forcing peak. We interpret this to be a result of the excitement of the fourth Rossiter mode close to the forcing frequency that, unlike the time-switching second and third modes, remains dominant in time. The structures of this mode appear to be somewhat weaker than those in Fig. 13 for the unforced flow.

Figure 16 presents the SPL spectrum, spectrogram, and average centerline image of the Mach 0.30 resonance suppressed with use of the PP with time-delay control. This flow has features very similar to the Mach 0.32 case in Fig. 14 except that the frequencies of the peaks above the third Rossiter mode do not correspond to any of the resonant frequencies predicted in Fig. 5 for the Mach 0.30 flow. Inspection of the spectrogram (Fig. 16b) suggests that the PP control with time delay induces a competition between sidebands of the original resonant tone $\mathrm{e}^{23}$ and a broad peak centered about the
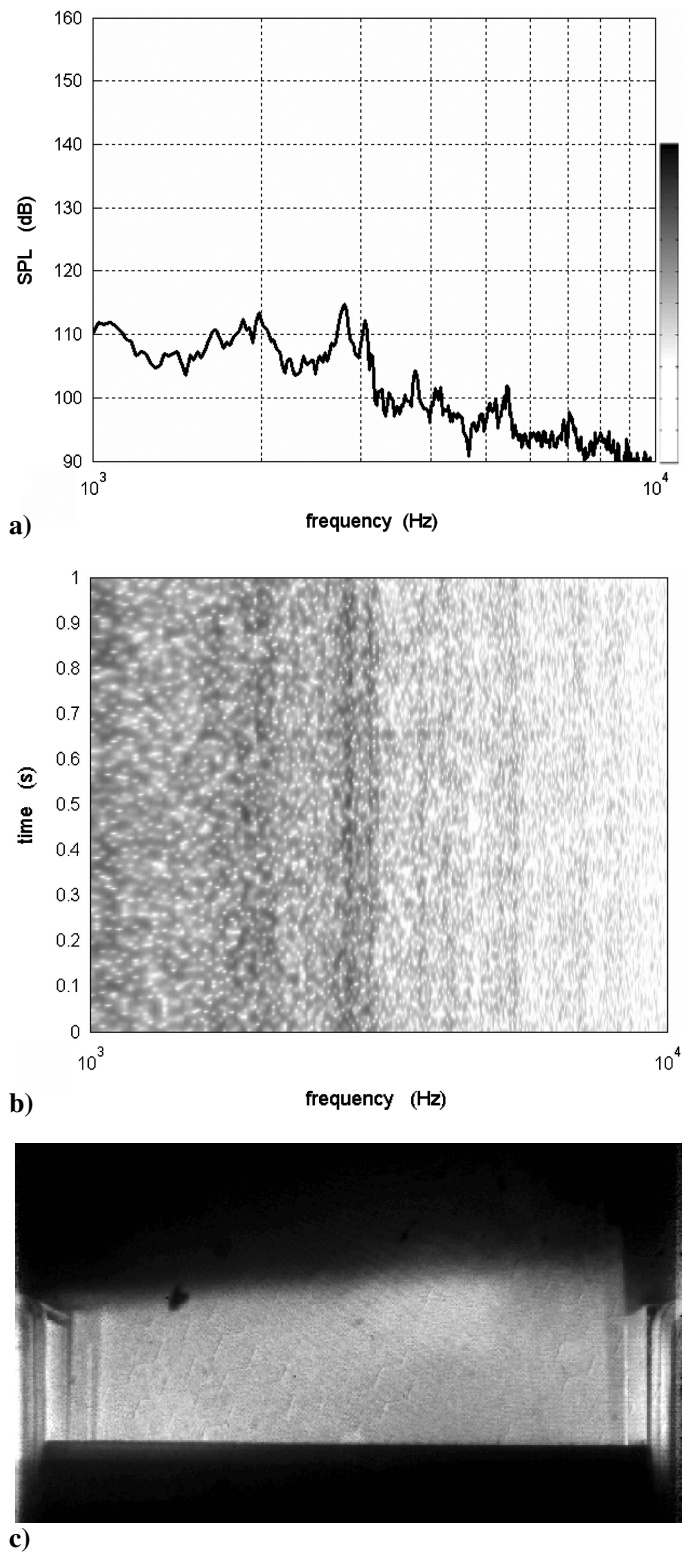

Fig. 16 Mach 0.30 flow with PP with time-delay control: a) SPL spectrum, b) spectrogram, and c) phase-locked average laser light-scattering image. 
second Rossiter mode. The spreading of the acoustic energy across the spectrum produces a quieter, broadband flow resembling the Mach 0.32 case. Similarly, the average centerline image of this flow is rather featureless due to the inability to lock the laser and camera to a steady peak.

\section{Conclusions}

We investigated closed-loop linear control for shallow cavity flows. Our experimental results confirmed that linear controllers, for example, $H^{\infty}$ controller, Smith predictor, and PID controller, derived from a single dominant-mode plant model are able to suppress the cavity oscillations at this frequency, but they shift the oscillations to another Rossiter frequency, which was not visible in the unforced case but could easily be excited, a phenomenon that has also been observed by other research groups using linear controllers. ${ }^{12,23,24}$

Adding a zero to the controller at these hidden Rossiter frequencies avoids this problem, and the resulting controller is very effective in reducing cavity noise without introducing strong additional tones. This controller was compared to a very effective open-loop method $^{14}$ for reducing cavity resonance and exhibited superior robustness with respect to departure of the Mach number from the design conditions. An additional benefit of the closed-loop control method is the lower power requirement to achieve comparable suppression of the resonance.

An interpretation is presented of the physical mechanisms by which the open-loop forcing and the closed-loop, PP with time-delay controller reduce the cavity flow noise. The results support the idea that these controls induce in the system a state similar to multimode resonance where rapid switching occurs between the resonant modes and the modes induced with forcing. The competition between the modes for the available energy that can be extracted from the mean flow produces a less efficient resonant system where no single mode locks in to dominate the spectrum.

An extension of this work is to consider switching of multiple linear models dependent on the Mach number, as well as the input applied. The long term goal of this research is to provide analytical linear/nonlinear models for the cavity flow dynamics and design feedback controllers accordingly.

\section{Acknowledgments}

This work is mainly supported by the U.S. Air Force Laboratory's Air Vehicle Directorate and the Air Force Office of Scientific Research through the Collaborative Center of Control Science (Contract F33615-01-2-3154) and partially supported by the DAGSI Fellowship Program and by the European Commission under Contract MIRG-CT-2004-006666. We thank the rest of the flow control team at the Collaborative Center for Control Science, James Myatt, James DeBonis, R. Chris Camphouse, Önder Efe, Edgar Caraballo, and James Malone. We thank David Williams and Louis Cattafesta for fruitful discussions.

\section{References}

${ }^{1}$ Doyle, J. C., Francis, B. A., and Tannenbaum, A. R., Feedback Control Theory, Macmillan, New York, 1992, Chaps. 10 and 12.

${ }^{2}$ Gad-el-Hak, M., Flow Control-Passive, Active, and Reactive Flow Management, Cambridge Univ. Press, New York, 2000.

${ }^{3}$ Cattafesta, L. N., III, Williams, D. R., Rowley, C. W., and Alvi, F. S., "Review of Active Control of Flow-Induced Cavity Resonance," AIAA Paper 2003-3567, June 2003.

${ }^{4}$ Samimy, M., Debiasi, M., Caraballo, E., Özbay, H., Efe, M. Ö., Yuan, X., DeBonis, J., and Myatt, J. H., "Closed-Loop Active Flow Control-A Collaborative Approach," AIAA Paper 2003-0058, Jan. 2003.
${ }^{5}$ Samimy, M., Debiasi, M., Caraballo, E., Özbay, H., Efe, M. Ö., Yuan, X., DeBonis, J., and Myatt, J. H., "Development of Closed-Loop Control for Cavity Flows," AIAA Paper 2003-4258, June 2003.

${ }^{6}$ Samimy, M., Debiasi, M., Caraballo, E., Malone, J., Little, J., Özbay, H., Efe, M. Ö., Yan, P., Yuan, X., DeBonis, J., Myatt, J. H., and Camphouse, R. C., "Exploring Strategies for Closed-Loop Cavity Flow Control," AIAA Paper 2004-0576, Jan. 2004.

${ }^{7}$ Siegel, S., Cohen, K., and McLaughlin, T., "Feedback Control of a Circular Cylinder Wake in Experiment and Simulation," AIAA Paper 2003-3569, June 2003

${ }^{8}$ Siegel, S., Cohen, K., and McLaughlin, T., "Feedback Control of a Circular Cylinder Wake in a Water Tunnel Experiment," AIAA Paper 2004-0580, Jan. 2004.

${ }^{9}$ Cohen, K., Siegel, S., and McLaughlin, T., "Sensor Placement Based on Proper Orthogonal Decomposition Modeling of a Cylinder Wake," AIAA Paper 2003-4259, June 2003.

${ }^{10}$ Cohen, K., Siegel, S., and McLaughlin, T., "Control Issues in ReducedOrder Feedback Flow Control,” AIAA Paper 2004-0575, Jan. 2004.

${ }^{11}$ Glauser, M. N., Higuchi, H., Ausseur, J., and Pinier, J., "Feedback Control of Separated Flows (Invited)," AIAA Paper 2004-2521, June 2004.

${ }^{12}$ Williams, D. R., Rowley, C. W., Colonius, T., Murray, R. M., MacMartin, D. G., Fabris, D., and Albertson, J., "Model-Based Control of Cavity Oscillations-Part 1: Experiments," AIAA Paper 2002-0971, Jan. 2002.

${ }^{13}$ Rowley, C. W., Williams, D. R., Colonius, T., Murray, R. M., MacMartin, D. G., and Fabris, D., "Model-Based Control of Cavity Oscillations Part II: System Identification and Analysis," AIAA Paper 2002-0972, Jan. 2002.

${ }^{14}$ Debiasi, M., and Samimy, M., "Logic-Based Active Control of Subsonic Cavity-Flow Resonance," AIAA Journal, Vol. 42, No. 9, 2004, pp. 1901-1909.

${ }^{15}$ Yuan, X., Efe, M. Ö., and Özbay, H., "On Delay-Based Linear Models and Robust Control of Cavity Flows," Advances in Time-Delay Systems, edited by S. Niculescu and K. Gu, Vol. 38, Lecture Notes in Computational Science and Engineering, Springer-Verlag, Berlin, 2004, pp. 287-298.

${ }^{16}$ Rowley, C. W., and Williams, D. R., "Control of Forced and SelfSustained Oscillations in the Flow past a Cavity," AIAA Paper 2003-0008, Jan. 2003.

${ }^{17}$ Foias, C., Özbay, H., and Tannenbaum, A. R., Robust Control of Infnite Dimensional Systems: Frequency Domain Methods, No. 209, Lecture Notes in Control and Information Systems, Springer-Verlag, Berlin, 1996.

${ }^{18}$ Hammond, J. K., and White, P. R., "The Analysis of Non-Stationary Signals Using Time-Frequency Methods," Journal of Sound and Vibration, Vol. 190, No. 3, 1996, pp. 419-447.

${ }^{19}$ Qian, S., and Chen, D., Joint Time-Frequency Analysis-Methods and Applications, Prentice-Hall, Upper Saddle River, NJ, 1996.

${ }^{20}$ Rossiter, J. E., "Wind Tunnel Experiments on the Flow Over Rectangular Cavities at Subsonic and Transonic Speeds," Royal Aircraft Establishment, RAE Technical Rept. 64037, 1964; also Aeronautical Research Council, Repts. and Memoranda 3438, Oct. 1964.

${ }^{21}$ Heller, H. H., and Bliss, D. B., "The Physical Mechanisms of FlowInduced Pressure Fluctuations in Cavities and Concepts for Their Suppression," AIAA Paper 75-491, March 1975.

${ }^{22}$ Yan, P., Debiasi, M., Yuan, X., Caraballo, E., Efe, M. Ö., Özbay, H., Samimy, M., DeBonis, J., Camphouse, C., Myatt, J. H., Serrani, A., and Malone, J., "Controller Design for Active Closed-Loop Control of Cavity Flows," AIAA Paper 2004-0573, Jan. 2004

${ }^{23}$ Cabell, R. H., Kegerise, M. A., Cox, D. E., and Gibbs, G. P., "Experimental Feedback Control of Flow Induced Cavity Tones," AIAA Paper 2002-2497, June 2002.

${ }^{24}$ Williams, D. R., Fabris, D., Iwanski, K., and Morrow, J., "Closed-Loop Control in Cavities with Unsteady Bleed Forcing," AIAA Paper 2000-0470, Jan. 2000.

${ }^{25}$ Cattafesta, L. N., III, Garg, S., Choudhari, M., and Li, F., "Active Control of Flow-Induced Cavity Resonance," AIAA Paper 97-1804, June 1997.

J. Gore

Associate Editor 